\title{
Outcome after spinal cord lesion: relation to country, social support, education, income and ways to measure outcome
}

\author{
JJ Wyndaele, Editor-in-Chief \\ Antwerp University Hospital, Antwerp, Belgium \\ E-mail: spinalcord@uza.be
}

Dear Spinal Cord reader,

This August issue of Spinal Cord starts with a profile of our president, Professor Wagih El Masry, who is a great figure head in spinal cord medicine.

Several papers in this issue deal with outcome, but outcome after treatment of a medical condition/rehabilitation can mean many different things. First, there are the direct health-related outcomes - what are the eventual complications and the causes of readmission? The outcome in quality of life of the individual and of those who care for him is also important. Another aspect may be ethical. Employment can also be affected by SCI, and this can result in changes in economic status. Listing limitations or abilities considers outcome from a different angle. So outcome can be measured in many ways, and can be investigated from a specific angle or using a combination of many metrics.

SCOPE (Spinal Cord Outcomes Partnership Endeavor) is a broad-based international consortium of scientists and clinical researchers who represent academic institutions, industry, government agencies, not-for-profit organizations and foundations. In their review, they assessed current and evolving tools for evaluating human spinal cord injury (SCI) outcomes for both clinical diagnosis and clinical research studies. They used a frame work for the appraisal of evidence of metric properties to examine outcome tools or tests for accuracy, sensitivity, reliability and validity for human SCI. More specifically, imaging, neurological, functional, autonomic, sexual health, bladder/bowel, pain, and psycho-social tools were evaluated. Several specific tools for human SCI studies have been or are being developed to allow the more accurate determination of whether a clinically meaningful benefit (improvement in functional outcome or quality of life) has been achieved as a result of a therapeutic intervention. They conclude that significant progress has been made, but further validation studies are required to identify the most appropriate tools for specific targets in a human SCI study or clinical trial.

Risk of mortality is also part of outcome. Krause and Carter found that apart from biographic and injury-related factors, low income and general social support have a role. They conclude that environmental factors are important predictors of mortality after SCI.

Outcome can also depend on how treatment is given. Four spinal rehabilitation units-in Denmark, Russia, Lithuania and Israel-looked into their unit's properties, rehabilitation objectives, ASIA scale, SCIM assessments and patient data. In the participating units, rehabilitation outcomes depend on SCI severity and unit-specific properties. They describe the effects of a moderately delayed rehabilitation with a long of stay in rehabilitation, and of early or slightly delayed rehabilitation combined with a shorter stay in rehabilitation.

One other factor that determines outcome is re-hospitalization. A large study from Ontario in Canada found that re-hospitalization occurred in $27.5 \%$ of individuals 1 year after initial, acute care discharge. Factors significantly associated with 1-year re-hospitalisation were length of stay, rural residence, more than 50 outpatient physician visits and more than 50 specialist visits following the index admission. The main causes of re-hospitalization were musculoskeletal, respiratory, gastrointestinal and urological disorders. The impact of such a high number of readmissions is obvious for both care and health economics.

A study in two Veteran Affairs Centres have looked into bloodstream infections acquired in hospital, from health care contact outside the hospital and in the community. These have similarities, but do differ in medical characteristics and causal micro-organisms. Treatment and management strategies may need to vary accordingly. 
A Japanese study made the interesting finding that in cases of complete sensorimotor paralysis due to cervical cord injury, early demonstration of patellar tendon reflex within 3 days after injury may offer an index to predict improvements in motor paralysis.

There are many, equally interesting studies in this issue: on dual energy X-ray Absorptiometry overestimation of bone mineral density of the lumbar spine; on levels of testosterone and gonadotrophins after long-standing tetraplegia; on testing of a protective device to protect from spinal cord and brain injury; on combined motor and somatosensory evoked potential monitoring for spine and spinal cord surgery; and on the relation between gut-specific autonomic testing and bowel dysfunction.

There is also a case report on fibro-cartilaginous embolism as cause of acute ischemic myelopathy.

Enjoy reading

Spinal Cord (2009) 47, 577-578; doi:10.1038/sc.2009.97 